\title{
A Compact Millimeter-wave Comb Generator for Calibrating Broadband Vector Receivers
}

\author{
Paul D. Hale, Kate A. Remley, Dylan F. Williams, Jeffrey A. Jargon, and C. M. Jack Wang \\ National Institute of Standards and Technology, Boulder, CO, 80305 USA \\ e-mail: hale@boulder.nist.gov
}

\begin{abstract}
We propose a compact and low-cost waveform source that can be used to calibrate the magnitude and phase response of vector receivers over a bandwidth as large as a few gigahertz. The generator consists of a broadband comb generator followed by a filter and amplifier. As a demonstration, we constructed and calibrated a source suitable for calibrating vector signal analyzers operating between $43 \mathrm{GHz}$ and 46.5 GHz. We then used the source to characterize the response of a commercially available vector signal analyzer. We compared the result with calibrations performed with a general-purpose source.
\end{abstract}

Index Terms-Calibration, comb generator, oscilloscope, vector signal analyzer, vector signal generator.

\section{INTRODUCTION}

B ROADBAND vector receivers are used to characterize signals that are modulated in phase as well as amplitude such as quadrature amplitude modulation (QAM). A simplified schematic of a vector signal analyzer (VSA, a type of vector receiver) is shown in Fig. 1. The VSA consists of an optional bandpass filter, a downconverter and an analog-todigital converter. These components cause linear and nonlinear distortion in the signal measurement as demonstrated in, e.g., [1] and [2]. Assuming the instrument is operated in a linear voltage regime, linear distortion dominates the measurement and can be quite significant [1], [2]. If the distortion can be characterized, then digital processing can be used in the VSA to compensate/calibrate the measurement system.

VSA calibration at microwave frequencies is often accomplished by internal calibration routines, however at millimeterwave frequencies, calibration can be more of an issue. In this work we propose and demonstrate a compact, low-cost waveform generator that can be used to calibrate the response of vector receivers operating in the millimeter-wave frequency regime.

A modulated signal source can be used to calibrate a VSA by assuming that the response of the source is ideal. However, modulated sources can exhibit significant phase errors that increase with modulation bandwidth, as in eg., [3][6]. Alternatively the source can be calibrated by use of a sampling oscilloscope and then used to calibrate the vector receiver [5]. However, most currently available general-purpose modulated sources are based on arbitrary waveform generators

U.S. Government work not protected by U.S. copyright

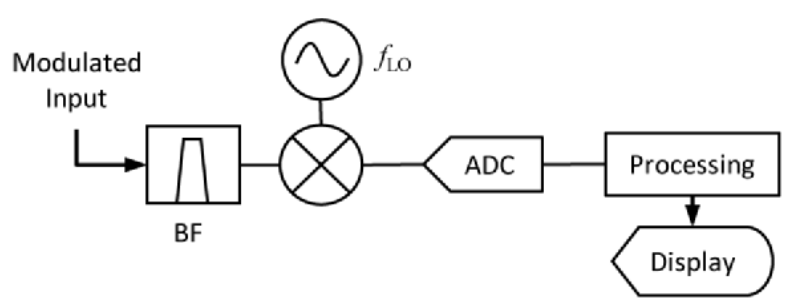

Fig. 1. Simplified schematic of a typical vector signal analayzer consisting of an optional bandpass filter (BF), a downconverter (mixer) and an analogto-digital converter (ADC). The output of the ADC is digitally processed to form, for example, a constellation diagram or a plot of magnitude and phase verses frequency.

and frequency converters. These are typically large, expensive, and susceptible to temperature changes and mechanical shock, making them impractical for field applications such as selfcalibration of a VSA.

Comb generators are often used for calibrating large-signal network analyzers (LSNAs). Since they can be based on a variety of nonlinear circuits, they are relatively simple, compact, and inexpensive, but are not as versatile as arbitrary waveform generator-based multisine sources. Some comb generators are based on step-recovery diodes and are sensitive to changes in drive power [7], [8] and temperature [9]. Recently, more stable comb generators have been demonstrated, e.g. [8], [10]. These generators produce impulse-like periodic pulses with a broad spectrum.

Because the energy in a train of impulse-like pulses is localized in a very short time interval, the power $P_{k}$ in the $k$ th tone of the comb is quite low for a given peak voltage $V_{p}$. For example, if the pulse repetition frequency is $f_{0}$ and the pulse is approximated as rectangular with duration $\Delta, P_{k}$ is given by

$$
\begin{aligned}
P_{k} & =\frac{V_{p}^{2} f_{0}^{2}}{50 \Omega}\left(\int_{-\Delta / 2}^{\Delta / 2} \cos \left(2 \pi k f_{0} t\right) d t\right)^{2} \\
& \leq \frac{\left(V_{p} f_{0} \Delta\right)^{2}}{50 \Omega}
\end{aligned}
$$

so that $P_{k} \leq-81 \mathrm{dBm}$ for typical parameters $V_{p}=0.1$ $\mathrm{V}, f_{0}=10 \mathrm{MHz}$, and $\Delta=20 \mathrm{ps}$. The noise background of a typical VSA is on the order of $-100 \mathrm{dBm}$ or more, giving only a $20 \mathrm{~dB}$ dynamic range without averaging. (A dynamic range on the order of $40 \mathrm{~dB}$ is required to obtain 


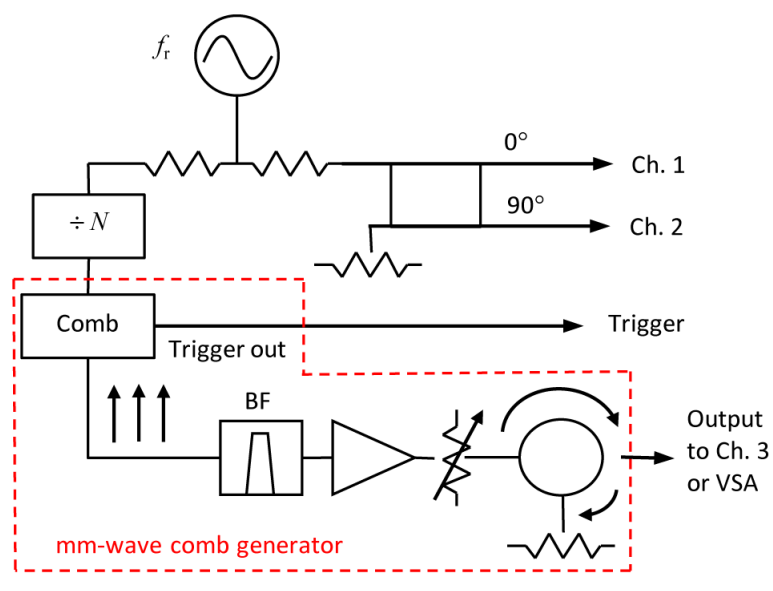

Fig. 2. Schematic drawing of the portable millimeter-wave comb generator and system used to characterize its output with known accuracy. Ports labeled Ch. 1, 2, and trigger refer to connections to a calibrated sampling oscilloscope that is used to calibrate the output of the generator, measured on channel 3. Channels 1 and 2 measure sine waves that are used by the NIST TBC algorithm [13] to correct the oscilloscope timebase. Once calibrated, the comb generator's output can be used to calibrate the linear response of a VSA.

error vector magnitude measurements that are accurate at the $\sim 1 \%$ level.) If the pulse duration could be increased, by use of band limiting and/or dispersive elements, while keeping the peak voltage fixed, the power in each tone would be increased, thus improving the signal-to-noise ratio when characterizing a vector receiver. Such an approach was hinted at in [10] and [5]. A source that was designed for a similar application over the $100 \mathrm{MHz}$ to $2 \mathrm{GHz}$ band was described in [11]. In this work we demonstrate a comb-generator-based source, with bandlimiting filter, operating between $43 \mathrm{GHz}$ and $46.5 \mathrm{GHz}$. With this source, other bands up to $67 \mathrm{GHz}$ are realizable.

\section{Comb Generator System For Millimeter-WAVE APPLICATIONS}

Our comb-generator system and the apparatus used to characterize its output are shown schematically in Fig. 2. We use commercially available components everywhere in the system. The box labeled "Comb" is a comb generator that is similar to that described in [8] but has been modified to have higher bandwidth and a $1.85 \mathrm{~mm}$ coaxial output connector. The output of the comb generator is filtered and temporally broadened by a $43 \mathrm{GHz}$ to $46.5 \mathrm{GHz}$ bandpass filter. Amplification and further filtering are provided by a $20 \mathrm{~dB}$ gain amplifier. An optional variable attenuator can be included for studying behavior of the vector receiver under test with respect to the signal power. The isolator is included to make the system source mismatch small and relatively constant when the reflection of the attenuator changes.

Different operating frequency ranges can be chosen by use of different filter/amplifier combinations. The highest useful frequency of our system, $67 \mathrm{GHz}$, is determined by the bandwidth and coaxial connector of the commercially available comb generator. Higher signal-to-noise ratios can be achieved by higher amplification and/or choosing a narrower bandwidth or a more dispersive filter, such as an all-pass filter [11].

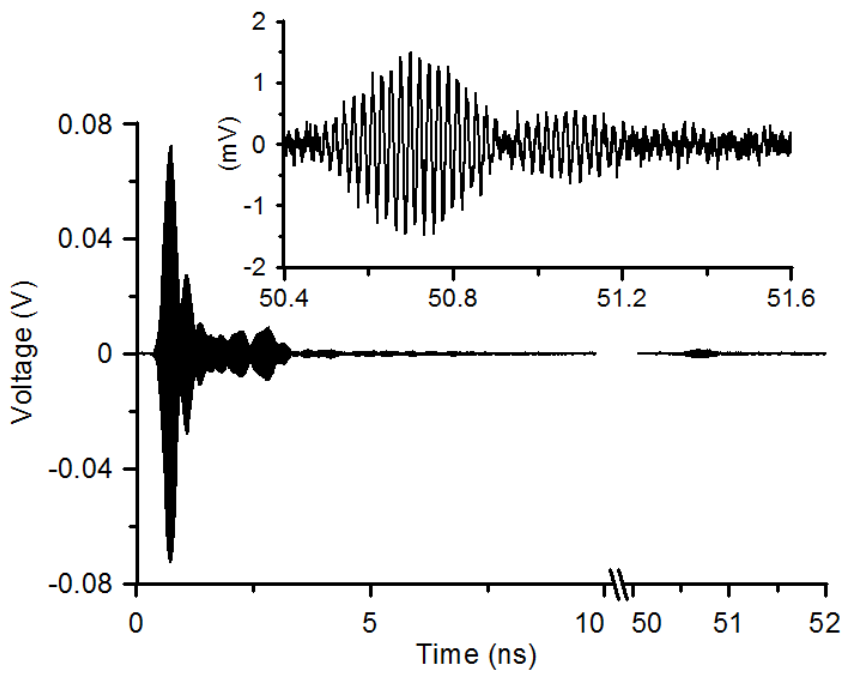

Fig. 3. Measured comb generator waveform.

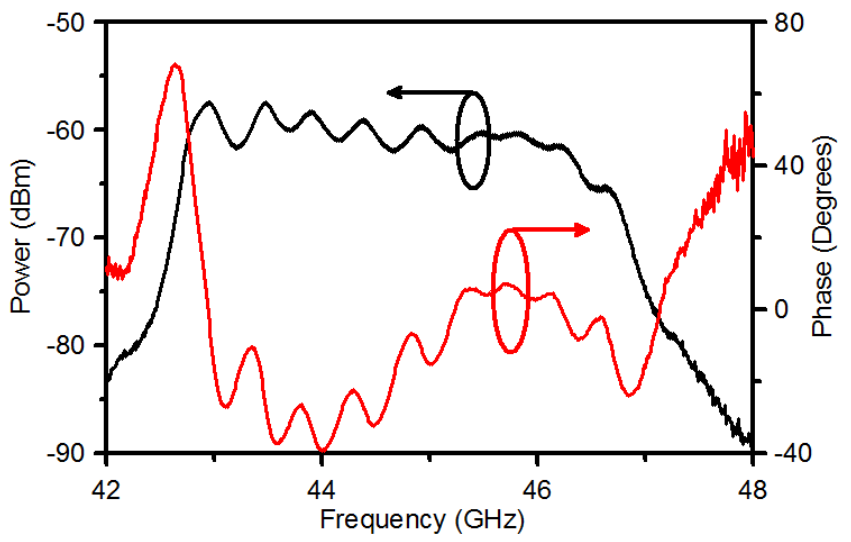

Fig. 4. Frequency domain view of comb generator waveform.

\section{Calibration of the Comb Generator System}

Calibration of the comb generator system is performed with a calibrated sampling oscilloscope [12] and by using procedures similar to those described in [3], [7], [13]. The system in Fig. 2 and our measurement procedures utilize the publicly available NIST timebase correction algorithm [13], [14]. The signal generator produces sine waves with frequency $f_{\mathrm{r}}$ on the order of $10 \mathrm{GHz}$ used for timebase distortion correction and as the reference for the trigger. The prescaler $(\div N$ block) produces a fast transition that triggers the comb generator, which is measured on channel 3 simultaneously with the sinusoid measurements on channels 1 and 2 . Because all of the samplers in the oscilloscope are activated by the same trigger pulse, the timing errors in all the channels in the oscilloscope are nearly identical [14]. The timebase correction algorithm fits the sinusoids measured on channels 1 and 2 and estimates the timing error in their measurement. This estimate is then used to correct the timing error in the measurement of the comb generator.

Portions of a waveform with repetition frequency $f_{0}=10$ $\mathrm{MHz}$ are shown in Fig 3. It is important to measure a full period of the waveform because temporally windowing will 
cause errors if energy is present away from the main pulse, as is the case in our comb generator system (as shown in Fig. 3). In our case, this extra pulse causes ripple in the spectrum with period of $20 \mathrm{MHz}$ and approximately $0.4 \mathrm{~dB}$ peak-to-peak magnitude variation (not resolved in Fig. 4) and 0.3 degree peak-to-peak phase variation.

A typical single waveform measurement is timebase corrected by use of the sequential timebase correction algorithm described in [13]. To facilitate further processing steps that use discrete Fourier transforms, the timebase corrected waveform is interpolated onto an evenly spaced temporal grid over an epoch equal to the period of the comb, that is, $1 / f_{0}$. Measurements of the comb are time consuming and subject to temperature drift. The drift error is efficiently corrected by first Fourier transforming the data and then using the phase-alignment algorithm of [15]. After drift correction, the waveforms are transformed back to the time domain and averaged. In our measurements the rms noise of a single waveform is approximately $1 \mathrm{mV}$ and is reduced to $0.1 \mathrm{mV}$ by averaging 100 measurements. Finally, the averaged measurements are corrected for the response of the oscilloscope and impedance mismatch, as described in [16], to obtain the response of the comb generator. Note that, while the samplers are calibrated by electro-optic sampling techniques through a National Metrology Institute, all other steps described above may be implemented by users.

\section{CAlibration OF A VSA AND COMPARison With Results ObTained With OTHER SOURCES}

We used our comb generator system to calibrate the response of a commercially available VSA over a bandwidth of $120 \mathrm{MHz}$ centered at $45 \mathrm{GHz}$. For comparison, we also synthesized two different multisines with $10 \mathrm{MHz}$ tone spacing centered at $45 \mathrm{GHz}$ [4]. These multisines were calibrated with the oscilloscope in the same way as with the comb generator system and had peak voltages that were comparable to that of the comb generator system. The first multisine had a (nominally) constant phase with respect to frequency and the second had Schroeder phase [17] characteristic.

The VSA was configured to acquire an integer number of cycles of the comb [18]. The input range was set about 4 $\mathrm{dB}$ below saturation. Multiple measurements of all signals were acquired, aligned using the technique of [15] and then averaged. Measurements of the comb generator system were aligned to a constant phase of zero.

Results of the measurements are shown in Fig. 5. The phase of the VSA measurements were compared to those measured by the calibrated oscilloscope. Agreement in the characterization of the VSA's phase error indicates that the portable comb system is performing on par with the rack of equipment that constitutes the AWG-based source. To quantify this, we used a paired $t$-test [19] to compare the mean measured phase $\bar{\phi}_{\mathrm{MS}}\left(f_{j}\right)=\left(\phi_{\mathrm{C}}\left(f_{j}\right)+\phi_{\mathrm{S}}\left(f_{j}\right)\right) / 2$ of the two multisine signals with the comb phase $\phi_{\mathrm{comb}}\left(f_{j}\right)$. The difference $d_{j}=\bar{\phi}_{\mathrm{MS}}\left(f_{j}\right)-\phi_{\mathrm{comb}}\left(f_{j}\right)$ averaged over the 13 frequencies is $\bar{d}=3 \times 10^{-5}$ and its standard deviation is $s_{\bar{d}}=0.042$. The test statistic $\bar{d} / s_{\bar{d}}=7.5 \times 10^{-4}$ produces

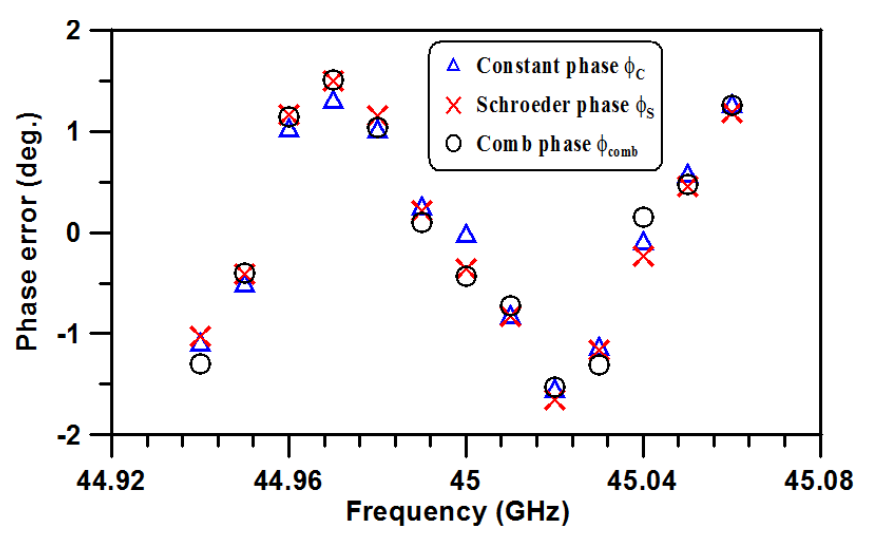

Fig. 5. Difference between phase measured with oscilloscope and phase measured with VSA based on measurements with the comb (o) and multisines with fixed phase $(\triangle)$ and Schroeder phase $(\times)$.

a $p$-value of 0.999 . Therefore, based on the data, we find no significant difference between the multisine- and comb generator-based calibrations.

\section{CONCLUSION}

We presented a straightforward measurement technique for calibrating vector signal analyzers at millimeter-wave frequencies by use of a calibrated comb generator. The method uses off-the-shelf parts and is portable, allowing users to conduct these calibrations in their own labs, without use of cumbersome, expensive AWG-based sources. The results presented here demonstrate that our prototype millimeter-wave comb generator can be used for calibrating VSAs without a significant degradation in the calibration of the phase response.

\section{ACKNOWLEDGEMENTS}

This work was partially supported by the Defense Advanced Research Projects Agency's Efficient Linearized All-Silicon Transmitter ICs (ELASTx) Program. The views, opinions, and/or findings contained in this article are those of the authors and should not be interpreted as representing the official views or policies, either expressed or implied, of the Defense Advanced Research Projects Agency or the U.S. Department of Defense.

\section{REFERENCES}

[1] C. J. Clark, A. A. Moulthrop, M. S. Muha, and C. P. Silva, "Transmission response measurements of frequency-translating devices using a vector network analyzer", IEEE Trans. Microw. Theory Tech., vol. 44, no. 12, pp.2724 -2737, Dec. 1996.

[2] D. F. Williams, H Khenissi, F. Ndagijimana, K. A. Remley, J. P. Dunsmore, P. D. Hale, C. M. Wang, and T. S. Clement, "Samplingoscilloscope measurement of a microwave mixer with single-digit phase accuracy," IEEE Trans. Microwave Theory Tech., Vol. 54, pp. 1210-1217, Mar. 2006.

[3] K. A. Remley, P.D. Hale, D.I. Bergman, D. Keenan,"Comparison of multisine measurements from instrumentation capable of nonlinear system characterization," 66th ARFTG Conf. Dig., Dec. 2005, pp. 34-43.

[4] K. A. Remley, P. D. Hale,D. F. Williams, J. Wang, "A precision millimeter-wave modulated-signal source", 2013 IEEE MTT-S Int. Microwave Symp., 2013.

[5] D. A. Humphreys, M. R. Harper, and M. Salter, "Traceable calibration of vector signal analyzers", 75th ARFTG Microwave Meas. Conf., 2010. 
[6] D. Rabijns, W. Van Moer, and G. Vandersteen, "Using multisines to measure state-of-the-art analog-to-digital converters," IEEE Trans. Instrum. Meas., vol. 56, no. 3, pp. 1012-1017, June 2006.

[7] H. C. Reader, D. F. Williams, P. D. Hale, and T. S. Clement, "Characterization of a $50 \mathrm{GHz}$ comb generator," IEEE Trans. Microwave Theory Tech., vol. 56, no. 2, pp. 515-521, Feb. 2008.

[8] D. Gunyan and Y.-P. Teoh, "Characterization of active harmonic phase standard with improved characteristics for nonlinear vector network analyzer calibration," 2008 IEEE MTT-S Int. Microwave Symp., pp. 73-79, 2008.

[9] J. A Jargon, J. D. Splett, D. F. Vecchia, and D. C. DeGroot, "An empirical model for the warm-up drift of a commercial harmonic phase standard," IEEE Trans. Instrum. Meas., vol. 56, no. 3, pp. 931-937, June 2007.

[10] D. B. Gunyan and J. B. Scott, U.S. Patent No. 7,423,470 B2, Sept. 9 , 2008.

[11] T. Van der Broeck, R. Pintelon, and A. Barel, "Design of a microwave multisine source using allpass functions estimated in the Richards domain,” IEEE Trans. Instrum. Meas., vol. 43, no. 5, pp. 753-757, Oct. 1994.

[12] T. S. Clement, P. D. Hale, D. F. Williams, C. M. Wang, A. Dienstfrey, and D. A. Keenan, "Calibration of sampling oscilloscopes with highspeed photodiodes," IEEE Trans. Microwave Theory Tech., vol. 54, pp. 3173-3181 (Aug. 2006).

[13] C. M. Wang, P. D. Hale, J. A. Jargon, D. F. Williams, and K. A. Remley, "Sequential estimation of timebase corrections for an arbitrarily long waveform," IEEE Trans. Instrum. Meas., vol. 61, no. 10, pp. 2689-2694, Oct. 2012.

[14] P. D. Hale, C. M. Wang, D. F. Williams, K. A. Remley, and J. Wepman, "Compensation of random and systematic timing errors in sampling oscilloscopes," IEEE Trans. Instrum. Meas., vol. 55, no. 6, pp. 21462154, Dec. 2006.

[15] K. A. Remley, D. F. Williams, D. Schreurs, G. Loglio, and A. Cidronali, "Phase detrending for measured multisine signals, 61st ARFTG Conf. Dig., June 2003, pp. 73-83.

[16] D. F. Williams, T. S. Clement, P. D. Hale, and A. Dienstfrey, "Terminology for High-Speed Sampling-Oscilloscope Calibration," 68th ARFTG Conference Digest, Boulder, CO, Nov. 30-Dec. 1, 2006.

[17] R. Pintelon and J. Schoukens, System Identification: A Frequency Domain Approach, New York, NY: IEEE Press, 2001.

[18] M. D. McKinley, K. A. Remley, M. Myslinski, and J. S. Kenney,"Eliminating FFT artifacts in vector signal analyzer spectra," Microwave Journal, vol. 49, no. 10, pp. 156-164, Oct. 2006.

[19] Peter W. M. John, Statistical Methods in Engineering and Quality Assurance, John Wiley \& Sons, 1990. 